\title{
Compassionate Treatment
}

National Cancer Institute

\section{Source}

National Cancer Institute. Compassionate Treatment. NCI Thesaurus. Code C71724.

Providing experimental therapies to very sick individuals even though they don't meet the critera for inclusion in a trial. 